\title{
Impact of the COVID19-related economic crisis and mitigation effects of social protection on HIV/AIDS and Tuberculosis: an integrated economic, mathematical and epidemiological modelling study
}

\section{Felipe Rubio}

Institute of Collective Health, Federal University of Bahia https://orcid.org/0000-0002-3428-5919

Alan Amad

Swansea University https://orcid.org/0000-0001-7709-5536

Erick Moya

Institute of Collective Health, Federal University of Bahia

Robson de Oliveira

Institute of Collective Health, Federal University of Bahia

Temidayo Aransiola

Institute of Collective Health, Federal University of Bahia

Rodrigo Anderle

Institute of Collective Health, Federal University of Bahia

Alberto Sironi

Institute of Collective Health, Federal University of Bahia

José Ordoñez

Institute of Collective Health, Federal University of Bahia

Mauro Sanchez

University of Brasília

Juliane de Oliveira

Centre for Data and Knowledge Integration for Health (CIDACS), Oswaldo Cruz Foundation

Luis Eugenio de Souza

Institute of Collective Health, Federal University of Bahia

Inês Dourado

Institute of Collective Health, Federal University of Bahia

James Macinko

Department of Health Policy and Management, University of California

Davide Rasella ( $\nabla$ davide.rasella@gmail.com)

Institute of Collective Health, Federal University of Bahia https://orcid.org/0000-0002-7260-4386 
Article

Keywords:

Posted Date: February 4th, 2022

DOI: https://doi.org/10.21203/rs.3.rs-1316131/v1

License: (c) (i) This work is licensed under a Creative Commons Attribution 4.0 International License. Read Full License 


\section{Impact of the COVID19-related economic crisis and mitigation effects of social protection on HIV/AIDS and Tuberculosis: an integrated economic, mathematical and epidemiological modelling study}

Felipe Alves Rubio ${ }^{1}$, Alan Alves Santana Amad ${ }^{2,4}$, Erick Delgado Moya ${ }^{1}$, Robson Bruniera de Oliveira ${ }^{1}$, Temidayo James Aransiola ${ }^{1}$, Rodrigo Volmir Anderle $^{1}$, Alberto Pietro Sironi ${ }^{1}$, José Alejandro Ordoñez ${ }^{1}$, Mauro Niskier

Sanchez $^{3}$, Juliane Fonseca de Oliveira ${ }^{2}$, Luis Eugenio de Souza ${ }^{1}$, Inês Dourado ${ }^{1}$, James Macinko ${ }^{5}$, Davide Rasella ${ }^{1,6}$

\footnotetext{
${ }^{1}$ Institute of Collective Health, Federal University of Bahia, Salvador, Brazil

${ }^{2}$ Centre for Data and Knowledge Integration for Health, Salvador, Brazil

${ }^{3}$ Department of Public Health, University of Brasilia, Brasilia, Brazil

${ }^{4}$ College of Engineering, Swansea University, Bay Campus, Swansea, UK

${ }^{5}$ Department of Health Policy and Management, University of California, California, USA

${ }^{6}$ ISGlobal, Hospital Clínic - Universitat de Barcelona, Barcelona, Spain
} 
The COVID-19 pandemic will have impactful long-term effects on the global rise of poverty and social inequalities, potentially compromising the achievement of SDGs for poverty-related diseases. We aimed to evaluate the impact of poverty increases, and the mitigation effects of social protection policies, on HIV/AIDS and Tuberculosis (TB) in Brazil, one of the largest and most unequal LMICs. We integrated economic, mathematical and epidemiological models to forecast the trends of HIV/AIDS and TB according to different future scenarios, showing that the implementation of social protection policies could mitigate long-lasting rises in poverty, avoiding -in the most pessimistic economic projections- an increase of $41 \%$ in the incidence 1 and $50 \%$ in the mortality from HIV/AIDS, and of $32 \%$ in the incidence and $1253 \%$ in the mortality from TB. Overall, more than 250 thousand cases and 343 thousand deaths from HIV/AIDS and TB could be averted up to 2030. 
The COVID-19 pandemic has been one of the most impactful and complex events in recent human history. Its burden will extend far beyond the COVID19 related diseases, and will encompass a long-term increase in morbidity and mortality due to the global rise in poverty and social inequalities [1]. 120 million people have already been pushed back into extreme poverty leading to an unprecedented rise in social vulnerability worldwide. The global poverty rate is projected to reach $7 \%$ by 2030 , and as a consequence, poverty-related diseases, that account for more than $45 \%$ of the disease burden in developing countries, will continue to spread among the more than 700 million individuals still living in extreme poverty [2]. This will halt and - in some Low and Middle-Income Countries (LMICs) reverse all progresses done for most poverty-related infectious diseases, in particular for Tuberculosis (TB) and AIDS [3, 4]. As emphasized in the WHO End TB Strategy, the most immediate and efficient way to reduce tuberculosis in the short and middle-term would be the expansion of social protection and universal health coverage [5]. Recent statistical models estimated that the elimination of extreme poverty would be able to reduce the global incidence of TB by $33 \%$ by 2035 [6]. Several studies have also shown the significant impact of poverty-reduction policies on HIV prevention [7], and the effects of conditional cash transfers on the reduction of the HIV/AIDS burden [8].

Brazil is one of the largest and most populous LMIC, and has also been one of the countries most dramatically affected by the COVID-19 pandemic [9]. Due to the resulting economic recession, poverty rates have suddenly increased all over the country. However, the prompt implementation in April 2020 of the Emergency Benefits Scheme (Auxilio Emergencial) (AE) had a strong impact on poverty rate, that reached in a few weeks its historic low of 4.6\% [10]. AE was launched to protect the new poor affected by the COVID-19 pandemic, and was initially limited to those not already covered by other social protection policies. By its volume of resources, coverage, timeliness, and impacts on income and poverty, it has been considered one of the soundest social protection responses worldwide. However, its abrupt interruption after a few months caused an increase of 329\% of poverty rates, that reached in the first trimester of 2021 an historical high of $16.1 \%$. AE was eventually reshaped with lower benefits and lower coverage, but while social vulnerabilities are still increasing in the country, effective Conditional Cash Transfers (CCTs) such the Programa Bolsa Familia (PBF) [11] have been terminated and fiscal austerity measures, implemented since the last economic crisis, have been maintained [12], conveying an uncertain future for the social protection in the country. Previous studies have already shown the mitigation effects of poverty-reduction policies on the increase of overall mortality during economic 
recessions [13], but their impact on poverty-related infectious diseases has never been evaluated. Due to these sudden changes in poverty rates, to the implementation of large-scale social protection policies such as the $\mathrm{AE}$, and to the uncertainty about future socioeconomic scenarios and policy responses, Brazil represents a natural experiment that could help us to evaluate the importance of mitigating the effects of the current global economic crisis in LMICs, and the consequences of not doing so. Moreover, the high burden of poverty-related infectious diseases in the country allows to assess the impact of such changing socioeconomic conditions on population health. As a matter of fact, Brazil has one of the highest TB burden worldwide, and although there have been improvements in the TB control in the last decades, the achievement of the Sustainable Development Goal (SDG) for TB is unlikely considering the current trends [14]. Regarding HIV/AIDS, while the effectiveness of the Brazilian response, based on the universalization of antiretroviral therapy in the 1990s and the current distribution-free pre-exposure prophylaxis, has been widely recognized, its incidence remains relatively high due to the heterogeneous distribution of socioeconomic conditions and risk factors in the country [15].

In this study, we have created an integrated economic, epidemiological and mathematical model to estimate the future impact of different economic crisis scenarios on the incidence and mortality from AIDS and TB, and the mitigation effect of alternative poverty-reduction policy responses up to 2030 in Brazil.

\section{Results}

Modelling poverty trends and poverty projections. Using data from national household surveys we estimated the time series of poverty rate in the country since 2003 (Figure 1): there was a steady reduction trend from the beginning of the period until the economic recession of 2014, when poverty started to increase until 2020, the first year of the pandemic. In this year, we used microdata on the individual income - from nationwide surveys implemented during the pandemic to estimate the real poverty levels with the introduction of the AE, shown in 2020 by the yellow and blue trajectories in Fig. 1. We also estimated the poverty rates trajectory if the AE would have not been implemented (green and red lines), subtracting the $\mathrm{AE}$ benefits from the household income in the individual microdata of the survey. After 2020, poverty rate were projected based on different simulation methods, and four possible economic scenarios up to 2030 were hypothesized: three deterministic scenarios (two pessimistic, one optimistic) developed by ap- 
plying exponential decay formulas calibrated to the empirical data of 2019-2020, and one derived by projections of autoregressive models applied to the previous poverty time series (Figure 1). The two pessimistic scenarios (green and red lines) illustrate how the lack of state intervention during the pandemic could cause a significant increase in the poverty rate for the following years, before an economic recovery which could occur in the medium term (in 2023 - green line, the less pessimistic scenario) or in the long term (in 2026, red line, the more pessimistic scenario). The more optimistic scenario (long term mitigation - blue line) show how, after the implementation of AE in 2020, hypothetical strong and sustained social protection interventions will be able to significantly reduce poverty rates, keeping them close to zero in the long term if maintained. The less optimistic scenario (mitigation for one year - yellow line) represent an intermediate scenario with the significant reduction of AE after 2020 (creating the rebound of poverty already observed in 2021) followed by the implementation of sustained social protection policies in the following years, able to continue the poverty-reduction trends of the previous decade.

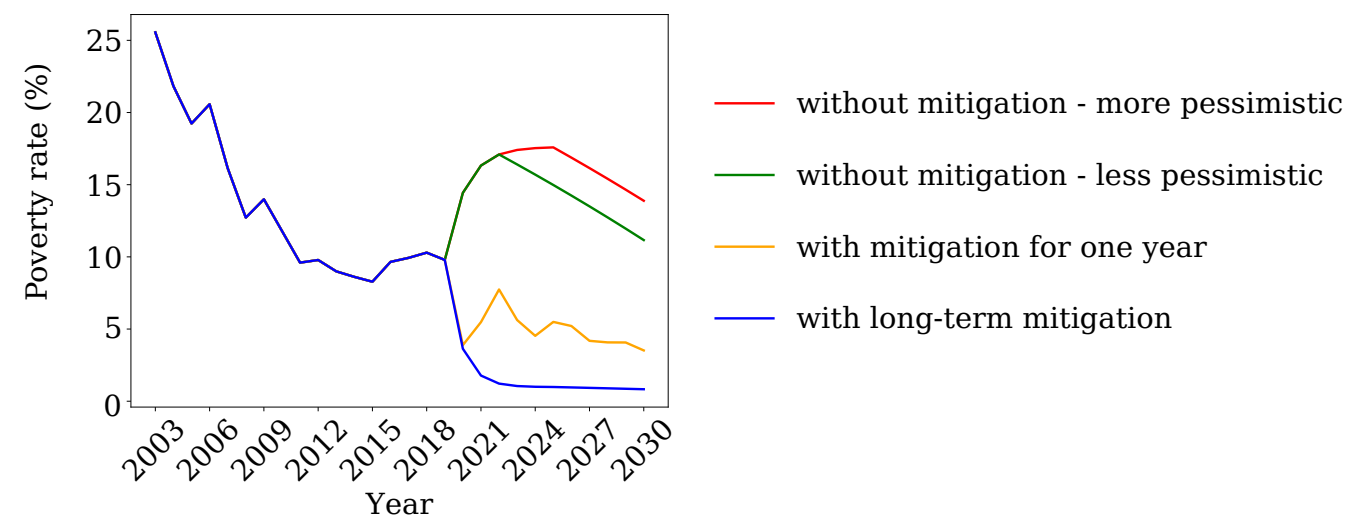

Fig. 1 Poverty rate scenarios between 2003 and 2030.

Mathematical modelling of HIV/AIDS and TB. The mathematical models dealing with HIV/AIDS and TB transmission dynamics are described in many papers [16, 17, 18, 19, 20]. However, the inclusion of social determinants of health, and in particular poverty, in epidemiological models is still barely addressed. Galanis and Hanieh (2021) discussed the inclusion of three social determinants of health into the modelling framework of infectious diseases, but only with as a theoretical 
approach [21]. In light of this, we developed two compartmental models composed by nonlinear differential equations for HIV/AIDS and for TB transmission dynamics to forecast the impact of the previously simulated economic scenarios on the trajectories of incidence and mortality from AIDS and TB in Brazil up to 2030.

The HIV/AIDS model was based on the work of Huo et al. (2016), which considers the population divided into five compartments - susceptible (S), HIVpositive individuals in the stage of HIV infection $(I)$, individuals with full-blown AIDS but not receiving ART treatment $(A)$, individuals being treated with undetectable viral load $(T)$, and individuals who have changed their sexual habits such that they are considerate immune to HIV infection by sexual contact $(R)$ [19]. Although it is a simplified model that contains only the main compartments and dynamics of the disease, it also considers important aspects such as the possibility that not everyone is susceptible to the HIV infection. The TB model was based on the work of Gomes et al. (2019), which presented a mathematical model composed of four nonlinear ordinary differential equations [20]. Here, the population is divided into Susceptible $(S)$, primary infection $(P)$, latent infection $(L)$, and active tuberculosis disease $(I)$. Figure 2 depicts the epidemiological scheme of the HIV/AIDS and TB models. Epidemiological meaning of each parameter model is given in the Methods section.
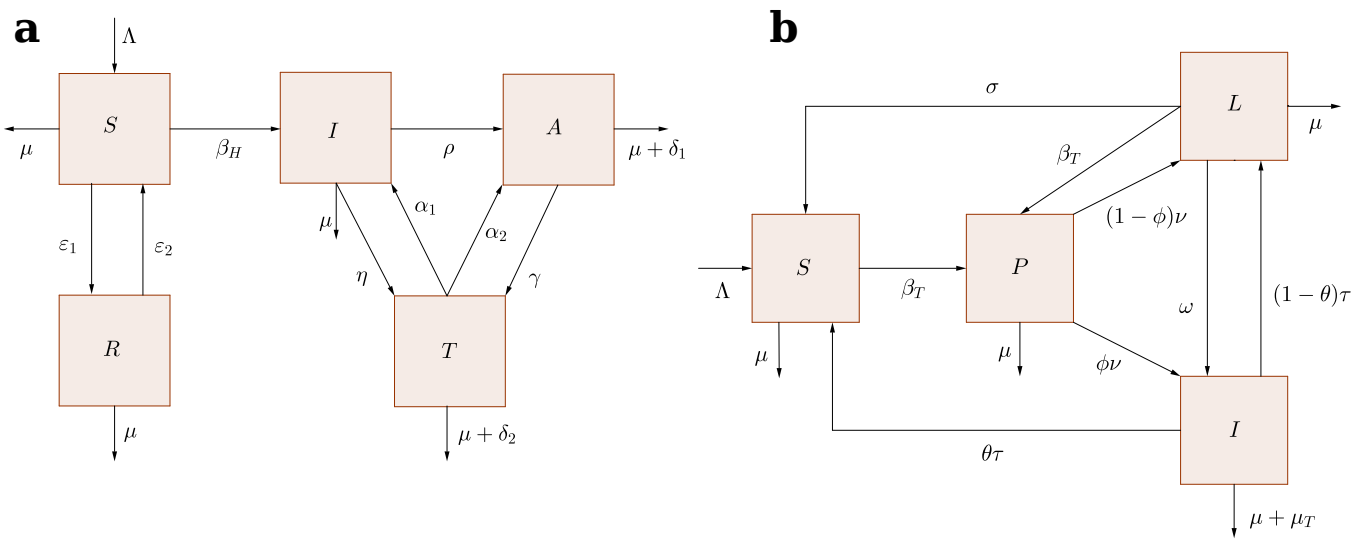

Fig. 2 Epidemiological scheme of the mathematical models. a. HIV/AIDS transmission model (1). b. Tuberculosis transmission model (2).

The calibration process, developed for both HIV/AIDS and TB up to 2019, was based on the simultaneous fitting of both the incident cases and the deaths estimated by the compartmental models with the official data of incidence and 
mortality from the Brazilian Ministry of Health. This strategy has been adopted to ensure reliable projections for both indicators of each disease. As a novel approach, during the calibration process, we included the time series of the annual poverty rate into all the parameters of the models (described in Methods), allowing to estimate how poverty was associated with effective contact rate, cure rate, case-fatality rates and all other parameters of HIV/AIDS and TB dynamic compartmental models. Poverty rate was selected for two reasons: first it is the social determinant of health with the strongest influence on TB and HIV/AIDS dynamics, second it is the socioeconomic indicator that had the largest variations over the last decade and during the current COVID-19 related crisis, and could be considered a proxy for other indicators.

Before the model calibration process, a sensitivity analysis of the models was carried out in order to assess which parameter most influenced the variation of the model variables over time. We found that these parameters were $\beta_{H}$ (force of the infection) for the HIV/AIDS model and $\nu$ (rate of progression from primary infection) for the TB model (Supplementary Information). Model (1) was fitted to the annual new AIDS cases and deaths reported by the Brazilian Ministry of Health between 2007 and 2019 [22]. Similarly, the TB model (2) was fitted to the annual new TB cases and deaths during 2003 and 2019 [23]. The initial year for calibration differs in the models due to the change in notification that occurred in 2007 for HIV/AIDS. We also did not consider the year 2020 due to the beginning of the COVID-19 pandemic and the related under-registration of HIV/AIDS and TB cases. Extended Data Table 1 shows the fitted values of the respective model parameters.

We estimate the HIV/AIDS and TB model parameters applying a multiobjective genetic algorithm [24], which is capable of involve more than one objective function to be optimized simultaneously, in our case, incidence and mortality in each model (see Methods for more details). Following the Brazilian population data [25], the natural birth and mortality rates, which are common parameters in both models, were assumed to take the values $\Lambda=4,468,962.55$ and $\mu=0.0132$, respectively, in order to fit the Brazilian total population data between the years 2003 and 2030 (Extended Data Fig. 1). Based on literature, parameter intervals were assumed to each model for the calibration process, (Tables 2 and 3 in Methods section).

Modelling HIV/AIDS and TB incidence and mortality rates. For calculating incidence and mortality rates from HIV/AIDS and TB, we divided the number of cases and deaths for each disease per 100,000 person-years (p-y), respectively. Figure 3 shows that, depending on the economic scenario, the incidence and mor- 
tality rates of AIDS and TB will follow remarkably different trends: the sudden increase of poverty rates, due to the economic crisis and the lack of social protection policies (without mitigation - more pessimistic, red line; and less pessimistic green line), will cause a considerable rise in TB incidence (Figure 35) and mortality (Figure 3d). While after a certain number of years, depending of the economic scenarios (red vs green lines), poverty rates will start to steadily decrease, TB incidence and - to a lesser extent - TB mortality will delay their trend reductions. In the more optimistic scenarios, TB incidence and mortality will tend to have trajectories very similar to poverty rates: in case of the $\mathrm{AE}$ mitigation implemented only for one year (yellow line), followed by sustained social policies, TB incidence and mortality will follow the trends of the previous decade, while in case of a long-term mitigation with extremely strong AE-like social policies (blue line), all TB indicators will decrease significantly more. The incidence (Figure $3 \mathrm{a}$ ) and mortality rates (Figure $3 \mathrm{~b}$ ) from AIDS will increase less quickly and abruptly than $\mathrm{TB}$, due to the different compartmental model dynamics and sensitivity to poverty rates, but the shape of the curves and difference between scenarios will be similar to TB.

Table 1 shows the incidence and mortality rate ratios - in different years of the study period - using the optimistic scenarios of 1-year AE poverty mitigation plus sustained social policies in the following years (yellow line) as reference values. This scenario was considered the most plausible if social protection will be preserved and strengthened in the country over the next years. While in the short and medium term the differences between scenarios will be stronger for TB, both for incidence and mortality (27\% and $45 \%$ more, respectively, in the most pessimistic scenario in 2025), in the long term (2030) rate ratios will be slightly larger for AIDS morbidity and mortality (41\% and 50\% more, respectively, in the most pessimistic scenario in 2030). In terms of the overall number of averted new infections and deaths (last two columns of Table 1) up to 2030, the number of averted new cases of TB over the period will be more than 3 times higher than for AIDS in all the scenario, reaching almost 190 thousand (against 64 thousand for AIDS) averted cases if the effect of the economic crisis would be mitigated during the acute phase of the recession and afterward. For the same scenarios comparison, the number of averted deaths will be considerably higher for AIDS (almost 25 thousand) than for TB (almost 18 thousand). More than 65 thousand new cases of TB and AIDS and more the 10 thousand of deaths will be averted if extremely strong AE-like social policies (blue line) will be implemented instead of the less optimistic scenario (yellow line). 
$\mathbf{a}$

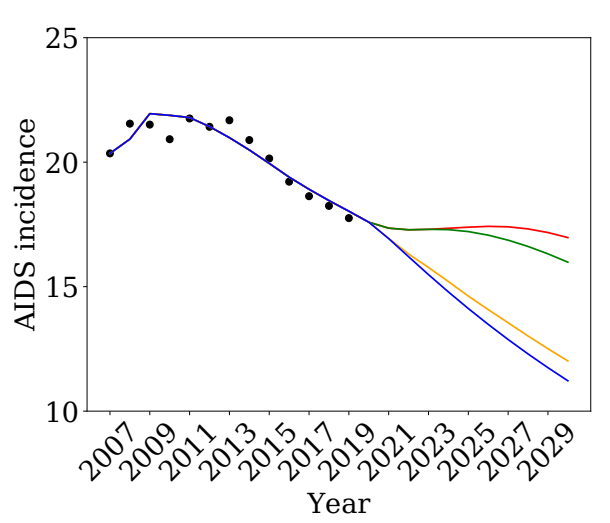

C

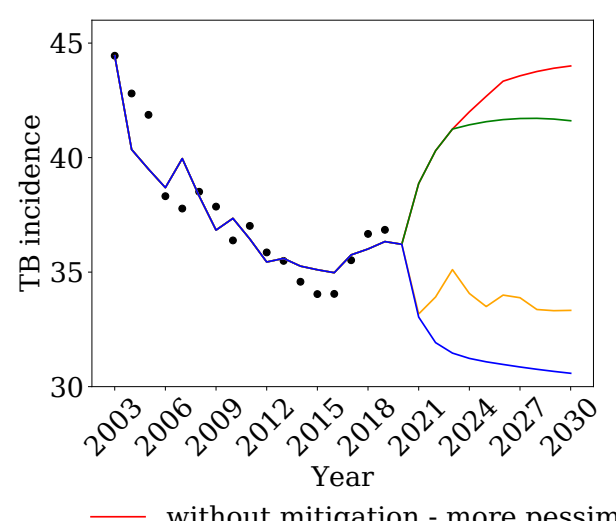

without mitigation - more pessimistic with mitigation for one year b

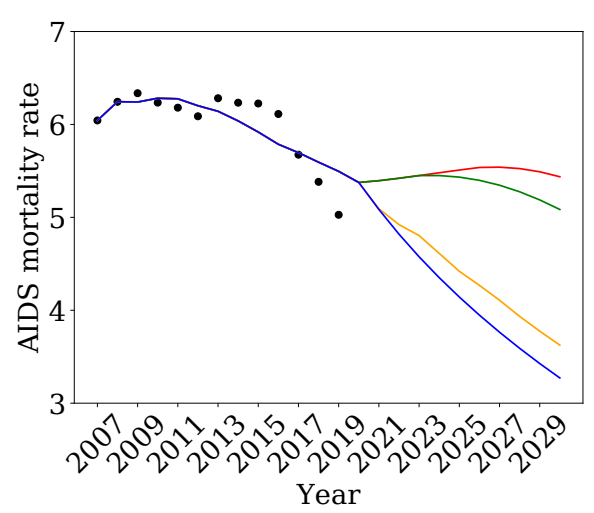

d

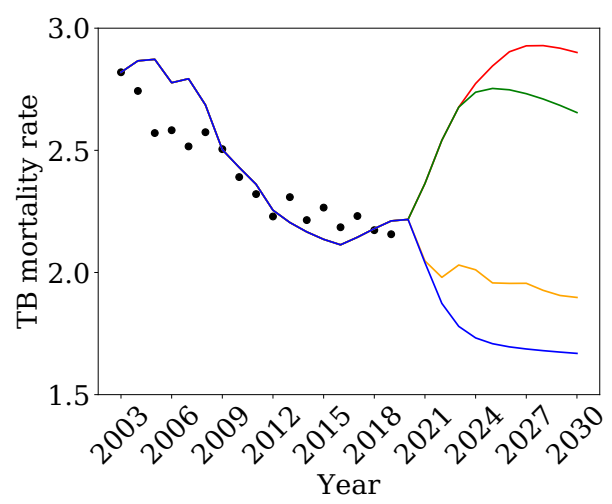

without mitigation - less pessimistic with long-term mitigation • Data

Fig. 3 AIDS and TB morbimortality. a. AIDS incidence rate under different scenarios of poverty rate between 2007 and 2030. b. AIDS mortality rate under different scenarios of poverty rate between 2007 and 2030. c. TB incidence rate under different scenarios of poverty rate between 2003 and 2030. d. TB mortality rate under different scenarios of poverty rate between 2003 and 2030. 
Table 1 Incidence and mortality rate ratios in specific years, and avoided cases and death over the all period, between the economic scenarios and the mitigation policy for one year as reference category.

\begin{tabular}{|c|c|c|c|c|c|c|c|c|c|}
\hline \multirow{3}{*}{ Economic crisis scenario } & & \multicolumn{6}{|c|}{ Rate ratio by year } & \multicolumn{2}{|c|}{$\begin{array}{l}\text { Cases and deaths } \\
\text { averted over the pe- } \\
\text { riod (n.) }\end{array}$} \\
\hline & & \multicolumn{2}{|c|}{2021} & \multicolumn{2}{|c|}{2025} & \multicolumn{2}{|c|}{2030} & \multirow[b]{2}{*}{ AIDS } & \multirow[b]{2}{*}{ TB } \\
\hline & & AIDS & TB & AIDS & TB & AIDS & TB & & \\
\hline \multirow{2}{*}{ Scenario 1 (higher) } & Incidence & 1.02 & 1.17 & 1.19 & 1.27 & 1.41 & 1.32 & 64,083 & 189,381 \\
\hline & Mortality & 1.06 & 1.15 & 1.25 & 1.45 & 1.50 & 1.53 & 24,811 & 17,877 \\
\hline \multirow{2}{*}{ Scenario 2 (medium) } & Incidence & 1.02 & 1.17 & 1.18 & 1.24 & 1.33 & 1.25 & 55,905 & 162,996 \\
\hline & Mortality & 1.05 & 1.15 & 1.23 & 1.41 & 1.40 & 1.40 & 21,820 & 15,257 \\
\hline \multirow{2}{*}{ Scenario 3 (lower) } & Incidence & 1.00 & 1.00 & 0.97 & 0.93 & 0.93 & 0.92 & 10,760 & 55,203 \\
\hline & Mortality & 1.00 & 1.00 & 0.94 & 0.87 & 0.90 & 0.88 & 5,711 & 4,691 \\
\hline
\end{tabular}

\section{Discussion}

In our study we have integrate economic, mathematical and epidemiological models to show for the first time, in one of the largest LMICs, the strong impact that the COVID19-related global economic crisis will have on the increase of HIV/AIDS and TB morbidity and mortality, and the important mitigation effects of a prompt and long-lasting implementation of social protection policies to counteract the rise in poverty rates. While during the COVID-19 pandemic all efforts have been focused on the control and elimination of its infectious agent, a large economic recession have hit the majority of LMICs, and its length and magnitude will profoundly affect the trends of all poverty-related diseases, in particular HIV/AIDS and TB. Relieving poverty should be the core of economic development since it directly reflects the well-being of the population. High poverty rates are for instance commonly indicative of high levels of unemployment, inequality, crime, food insecurity and malnutrition [26]. Brazil witnessed a persistently high rate of poverty the last century, before the economic and structural reform successfully implemented in the last two decades allowed the creation of a progressively larger welfare state [27]. Since then, Brazil has been one of the international references on poverty reduction until 2015 , when a large economic crisis hit the country. Social welfare programs such as CCTs targeted at the poor population and minimum wage valorization were mostly responsible for the reduction of poverty in Brazil from early 2000s [27, 28], whereas its increase from the year 2015 is attributed to the political and economic crisis that caused an expressive rise in un- 
employment [29]. This economic recession caused the increase of the number of Brazilians below the poverty line from about 17.0 to 23.3 million, meaning about 6.3 million "new poor" Brazilians [29]. The Covid-19 pandemic aggravated the already growing poverty rate trends as the employment of the poor and middleclass was mostly affected by the crisis [30]. While the AE was an important mitigating factor in the first phase of the crisis, this has been temporary since the value and coverage of the financial aid reduced steadily, whereas unemployment continued increasing $-11.8 \%$ to $13.8 \%$ from the year 2019 to 2020 [31]. Achieving the Sustainable Development Goal of zero poverty by the year 2030 appears to be remote if Brazil continues on the trend observed since the year 2015. Given the past experience, conditional cash transfer programs and financial aid for the poorest have been a successful interventions for reducing poverty rates and have also shown to be effective in mitigating the effect of the COVID-19 pandemic. Given the projections of this study, eradicating poverty by 2030 would be feasible only through the implementation of a broad and consistent welfare state focused on the most vulnerable populations.

While poverty has consistently been recognized as one of the most important risk factors for Tuberculosis [6], and poverty-reduction programs such as cash transfers have repeatedly shown a significant reduction in TB morbidity and mortality [29, 32, 33], for HIV/AIDS the evidence is weaker [8]. Poverty could affect housing conditions, overcrowding common spaces and decreasing ventilation in the rooms of the house, consequently increasing the possibility to contract TB through aerosol from infected individuals [34]. Regarding HIV/AIDS, poverty could force transactional sex and/or prostitution among the most vulnerable women, besides acting negatively on other mechanisms related to self-esteem and sexual behaviours [35, 36]. Moreover, poverty could threat household food security and worsen the nutritional status of the members of the family, which has a direct effect on the risk to pass from the latent stage of TB and HIV to the infectious status [37, 38]. Furthermore, poverty can negatively affect the access to diagnostic and treatment, both because the lack of money for transportation fares and the impossibility to take days-off at work due to the extreme economic vulnerability [39]. Moreover, poverty tend to reduce the time of schooling, that is an important protection factor for both HIV/AIDS and TB in several steps of the causal pathway [40, 41].

Mathematical models of infectious diseases or biological systems are extremely useful tools for performing in silico experiments and predicting how diseases or biological processes evolve over time under different scenarios [42]. However, a single mathematical model approach is unable to provide exact answers since 
these models need to have a restricted number of factors to analyze and obtain an understanding of the transmission dynamics. Therefore, the integration of different areas becomes crucial for the investigation of complex process or diseases since the introduction of methods or mechanisms from different areas can contribute significantly to an overview of the overall phenomenon.

Our models have limitations. First, only poverty was included as a social determinant of health because, for an integrative approach, it was necessary a reduced number of parameters and factors to calibrate the mathematical models. However it has to be considered that, during the current COVID19-related global economic recession and for the future years, the vulnerability indicator that will change the most will undoubtedly be the poverty rate. The influence of other social determinants of health less sensitive to economic shocks, together with the effects of the introduction of new diagnostic and therapeutic technologies, have not been included in the models because we focused on comparing scenarios based on changes in poverty and in poverty-mitigation policies, and not on projecting the future trends of HIV/AIDS and TB. We assume that all factors not included in our models, and that could have influenced such trends, would have affected all the scenarios in a similar way, and would not have significantly changed our HIV/AIDS and TB morbidity and mortality comparisons. Another limitation is the simplicity of our compartmental models for HIV/AIDS and TB that, although they have already been used in similar studies [19, 20], do not consider more complex disease status, differences in treatment compliance, TB resistance and multi-morbidity of HIV/AIDS and TB. However, such models can be considered reliable for modelling these infectious diseases in the Brazilian context, where treatment compliance is overall high, TB resistance is below $1 \%$ and HIV/AIDSTB-multi-morbidity below $10 \%$ [43]. The last limitation is related to the poverty projections: while the exponential decay curves have been calibrated on real data, and have been used in similar studies [12], they model a behaviour of the economical crisis, and the year of its ending, that is just assumed based on theoretical considerations, while the unpredictability of the pandemic could still affect future economic scenarios.

In conclusion, understanding and measuring complex phenomena - such as the COVID19-pandemic effects on socioeconomic and health outcomes - requires the integration of multidisciplinary models, combining methods from different fields such as economics, mathematics, and epidemiology. Using this comprehensive approach, we were able to show the importance of implementing strong social protection policies to avert a large increase in morbidity and mortality from HIV/AIDS and TB during the post-pandemic period in LMICs. Further efforts 
should be made to understand and estimate the impact of the current global recession on other poverty-related diseases.

\section{Methods}

HIV/AIDS transmission model. Our mathematical model for HIV/AIDS transmission dynamics is based on the work of Huo et al. (2016), which is composed of five dynamical variables, which are susceptible $(S)$, HIV-positive individuals in the stage of HIV infection $(I)$, individuals with full-blown AIDS but not receiving ART treatment $(A)$, individuals being treated with undetectable viral load $(T)$, and individuals who have changed their sexual habits such that they are considerate immune to HIV infection by sexual contact $(R)$ [19].

Susceptible individuals have a constant per-capita birth rate $\Lambda$ and a per-capita death rate $\mu$. These individuals can be infected in contact with HIV-positive individuals through the effective contact rate $\beta_{H}$. Besides, some susceptible individuals change their sexual habits at a per-capita rate $\varepsilon_{1}$, which we considered that they now have safe sexual habits and because of that they do not contribute to the HIV transmission. However, some individuals in the $R$ class can neglect their safe sexual habits and return to the susceptible compartment at a per-capita rate $\varepsilon_{2}$. HIVpositive individuals after a period $\rho^{-1}$ become individuals with full-blown AIDS but not receiving treatment. On the other hand, HIV-positive individuals receiving treatment after a period $\eta^{-1}$ reach a undetectable viral load. In addition, if the treatment is stopped, the individuals in the T class can increase their viral load and becoming infectious $(I)$ or even full-blown AIDS $(A)$ at a per-capita rate $\alpha_{1}$ and $\alpha_{2}$, respectively. We also considered that full-blown individuals at percapita rate $\gamma$ receiving treatment going to the compartment $T$; and individuals in $T$ or $A$ classes have a diseased-related death rate $\delta_{1}$ and $\delta_{2}$, respectively. The mathematical model is given by the following nonlinear system of ordinary differential equations

$$
\left\{\begin{array}{l}
\frac{d S}{d t}=\Lambda-\frac{\beta_{H}}{N} I S-\left(\varepsilon_{1}+\mu\right) S+\varepsilon_{2} R \\
\frac{d I}{d t}=\frac{\beta_{H}}{N} I S+\alpha_{1} T-(\mu+\rho+\eta) I \\
\frac{d A}{d t}=\rho I+\alpha_{2} T-\left(\mu+\delta_{1}+\gamma\right) A \\
\frac{d T}{d t}=\eta I+\gamma A-\left(\alpha_{1}+\mu+\delta_{2}+\alpha_{2}\right) T \\
\frac{d R}{d t}=\varepsilon_{1} S-\left(\varepsilon_{2}+\mu\right) R
\end{array}\right.
$$


with $S(0)>0, I(0) \geq 0, A(0) \geq 0, T(0) \geq 0, R(0) \geq 0$, and $N$ represents the total population, which is given by $N=S+I+A+T+R$. Table 2 describes the nonnegative parameters of the system (1).

Table 2 Parameter description of the HIV/AIDS model (1).

\begin{tabular}{|c|c|c|c|}
\hline Parameter & Description & Value & Reference \\
\hline$\Lambda$ & Birth rate & $4,468,962.55$ & [25] \\
\hline$\mu$ & Natural mortality rate & $1 / 75.50$ & [25] \\
\hline$\varepsilon_{1}$ & $\begin{array}{l}\text { Rate of susceptible individuals who } \\
\text { changed their habits }\end{array}$ & $(0.029,0.031)$ & [19] \\
\hline$\varepsilon_{2}$ & $\begin{array}{l}\text { Rate of individuals who changed } \\
\text { their habits and return to the suscep- } \\
\text { tible compartment }\end{array}$ & $(0,0.0031)$ & [19] \\
\hline$\beta_{H}$ & HIV transmission rate & $(0,10)$ & Assumed \\
\hline$\rho$ & Progression rate to A from I & $(0.066,0.1)$ & [44] \\
\hline$\eta$ & Progression rate to $\mathrm{T}$ from I & $(2,4)$ & [45] \\
\hline$\alpha_{1}$ & $\begin{array}{l}\text { Treatment failure rate ( } \mathrm{T} \text { to I com- } \\
\text { partmental) }\end{array}$ & $(0,0.4)$ & $46,47,48,49$ \\
\hline$\alpha_{2}$ & $\begin{array}{l}\text { Treatment failure rate ( } \mathrm{T} \text { to } \mathrm{A} \text { com- } \\
\text { partmental) }\end{array}$ & $(0,0.4)$ & [46, 47, 48, 49 \\
\hline$\delta_{1}$ & $\begin{array}{l}\text { AIDS-related death rate (individu- } \\
\text { als with full-blown Aids) }\end{array}$ & $(0,0.1)$ & [50] \\
\hline$\delta_{2}$ & $\begin{array}{l}\text { AIDS-related death rate for individ- } \\
\text { uals being treated }\end{array}$ & $(0,0.05)$ & [50] \\
\hline$\gamma$ & Progression rate to $\mathrm{T}$ from $\mathrm{A}$ & $(0.2,2)$ & {$[51,45$} \\
\hline
\end{tabular}

Tuberculosis transmission model. We based on the work of Gomes et al. (2019), which presented a mathematical model composed of four nonlinear ordinary differential equations [20]. The population is divided into Susceptible $(S)$, primary infection $(P)$, latent infection $(L)$, and active tuberculosis disease $(I)$. We assumed that only susceptible individuals have a birth rate $\Lambda$ but all compartments have a natural death rate $\mu$. In contact with an infectious individual $(I)$, representing by the parameter $\beta_{T}$ the susceptible individual comes into a primary infection. After a period $\nu^{-1}$ individuals in the compartment $P$, a fraction $\phi$ acquires active tuberculosis, while a fraction $(1-\phi)$ moves to the latent compartment. Active tuberculosis individuals have an additional mortality rate $\mu_{T}$ due to the tuberculosis disease. We considered that through treatment representing by the per-capita rate $\tau$, a fraction $\theta$ completely eliminate the bacteria, which makes this individual return to the susceptible compartment. On the other hand, a fraction $(1-\theta)$ cannot eliminate the bacteria and becomes latent. These individuals in the latent compartment can become infectious in contact with infectious individuals $I$ or through reactivation return to the compartment $I$ at a per-capita rate $\omega$. We also assume that latent individuals can return to the susceptible compartment at a per-capita rate $\sigma$. 


$$
\left\{\begin{array}{l}
\frac{d S}{d t}=\Lambda-\mu S-\frac{\beta_{T}}{N} I S+\theta \tau I+\sigma L \\
\frac{d P}{d t}=\frac{\beta_{T}}{N}(I S+L I)-(\nu+\mu) P \\
\frac{d I}{d t}=\phi \nu P+\omega L-\left(\mu_{T}+\mu+\tau\right) I \\
\frac{d L}{d t}=(1-\theta) \tau I+(1-\phi) \nu P-\frac{\beta_{T}}{N} L I-(\omega+\mu+\sigma) L
\end{array}\right.
$$

with nonnegative initial conditions and $N$ is the total population defined by $N=S+P+I+L$. The nonnegative parameters of the system (2) are described in Table 3 .

Table 3 Parameter description of the TB model (2).

\begin{tabular}{|c|c|c|c|}
\hline Parameter & Description & Value & Reference \\
\hline$\Lambda$ & Birth rate & $4,468,962.55$ & [25] \\
\hline$\mu$ & Natural mortality rate & $1 / 75.50$ & [25] \\
\hline$\beta_{T}$ & TB transmission rate & $(0,50)$ & Assumed \\
\hline$\theta$ & $\begin{array}{l}\text { Proportion clearing infection upon } \\
\text { treatment }\end{array}$ & $(0.75,0.85)$ & [52, 53] \\
\hline$\nu$ & $\begin{array}{l}\text { Rate of progression from primary } \\
\text { infection }\end{array}$ & $(2,4)$ & [20] \\
\hline$\phi$ & $\begin{array}{l}\text { Proportion progressing from pri- } \\
\text { mary infection to active disease }\end{array}$ & $(0.01,0.1)$ & [20, 54, 55, 56] \\
\hline$\tau$ & Rate of successful treatment & $(0.66,2)$ & {$[20,52,53]$} \\
\hline$\omega$ & $\begin{array}{l}\text { Rate of reactivation of latent infec- } \\
\text { tion }\end{array}$ & $(0.001,0.1)$ & {$[20,54,55,56$} \\
\hline$\mu_{T}$ & TB-related death rate & $(0.03,0.08)$ & [43] \\
\hline$\sigma$ & Progression rate to $\mathrm{S}$ from $\mathrm{L}$ & $(0.001,0.05)$ & Assumed \\
\hline
\end{tabular}

Incorporating poverty in the mathematical modelling. Models (1) and (2) capture the dynamics of HIV and Tuberculosis transmission in a simplified way. However, social determinants significantly impact incidence and mortality rates of these diseases. Accordingly to the work of Galanis and Hanieh (2021), here we assumed a linear form to include the effect of economical aspects in some model parameters through the annual poverty rate [21], that is, a biological component characterized by the constant term and a linear term characterized by the influence of the annual poverty rate. For the HIV/AIDS model (1), the parameters influenced by the poverty rate are 


$$
\begin{aligned}
\beta_{H}(t) & =\beta_{H 1}+\beta_{H 2} p(t) \\
\delta_{1}(t) & =\delta_{11}+\delta_{12} p(t) \\
\delta_{2}(t) & =\delta_{12}+\delta_{22} p(t) \\
\alpha_{1}(t) & =\alpha_{11}+\alpha_{12} p(t) \\
\alpha_{2}(t) & =\alpha_{12}+\alpha_{22} p(t) \\
\rho(t) & =\rho_{1}+\rho_{2} p(t) \\
\eta(t) & =\eta_{1}-\eta_{2} p(t) \\
\gamma(t) & =\gamma_{1}-\gamma_{2} p(t) \\
\varepsilon_{1}(t) & =\varepsilon_{11}-\varepsilon_{12} p(t) \\
\varepsilon_{2}(t) & =\varepsilon_{21}+\varepsilon_{22} p(t)
\end{aligned}
$$

where $0<p(t)<1$ represents the proportion of poor individuals in year $t$. Based on the literature, we assumed a positive relationship of the poverty rate with all parameters except $\eta, \varepsilon_{1}$, and $\gamma$.

For the TB model (2), we assume that the following parameters are influenced by the poverty rate, and are defined as

$$
\begin{aligned}
\beta_{T}(t) & =\beta_{T 1}+\beta_{T 2} p(t) \\
\nu(t) & =\nu_{1}+\nu_{2} p(t) \\
\tau(t) & =\tau_{1}-\tau_{2} p(t) \\
\omega(t) & =\omega_{1}+\omega_{2} p(t) \\
\mu_{T}(t) & =\mu_{T 1}+\mu_{T 2} p(t)
\end{aligned}
$$

Therefore, in order to incorporate the poverty rate influence, we include the parameter formulation given by (3) to (17) in the models (1) and (2).

Forecast and scenarios of poverty rate. Data. We calculate poverty rates using three National Household Surveys (PNAD) - the regular PNAD, Continuous-PNAD, and PNAD-COVID are used for 2001-2011, 2012-2019, and 2020, respectively. These surveys are representative of the entire Brazilian population and are similar in structure, although they differ in terms of sample size and collection strategy. The benefit values and eligibility criteria of the Bolsa Família cash transfer program (PBF) are obtained from official documents published by the Ministry of Social Development (MDS).

Poverty Measurement Method. Poverty lines are monetary values used to determine the percentage of a population below specific levels of economic wellbeing, measured using income levels. In this study, poverty rate in year $t$ is measured as the proportion of individuals that earn per capita income below the poverty line stipulated as the eligibility condition for participation in the PBF, Poor $_{t}$, compared to the total Brazilian population, Pop, i.e. Poor $_{t} /$ Pop $_{t}$. The poverty rate is obtained for the year 2010 by linear interpolation due to the lack of household surveys since the national Census was conducted in the same year.

Autoregressive approach. The poverty rates calculated from the year 2001 to 2020 are used to forecast values from 2021 to 2030 using Vector Autoregression (VAR) models represented as 


$$
Y_{t}=\beta_{0}+\beta_{1} Y_{t-k}+\text { trend }+\varepsilon_{t}
$$

where the dependent variable, $Y_{t}$, is the poverty rate for the year $t$, and the regressors are the $k$ lagged values of poverty rate, $Y_{t-k}$, and a trend, trend; $\varepsilon_{t}$ is the error term that is assumed to be a 'white noise' [57].

Deterministic approach. Besides the autoregressive approach, we perform the forecast of poverty rate using an exponential growth function. This deterministic approach permits the flexible creation of a wide range of poverty scenarios and growth tendencies using specified parameters. The scenarios created in this study are grouped into two major categories - optimistic (where the poverty rate reduces) and pessimistic (where the poverty rate increases). Optimistic scenarios are calculated using the following equation

$$
\operatorname{Pov}_{t}=\operatorname{Pov}_{t 0}-C_{i} \operatorname{Pov}_{t 0}\left(1-e^{-K_{i} t}\right)
$$

whereas the pessimistic scenario is expressed as

$$
\operatorname{Pov}_{t}=\operatorname{Pov}_{t 0}+C_{i} \operatorname{Pov}_{t 0}\left(1-e^{-K_{i} t}\right)
$$

Although the optimistic and pessimistic scenarios are simply mirrored equations, the behaviors of the projected trends depend on the values of the parameters detailed below

- $t$ - time variable, whereby $t=\{2001,2002, \ldots, 2030\}$ and $t 0$ is the baseline or reference year;

- $i$ - identifies the moments of growth. In this study, we formulate two moments, i.e $t=$ $\{1 ; 2\}$ - the first moment of high growth and a second moment of low and steady growth. The time duration of these moments $t$ is specified randomly and can be adjusted;

- $\mathrm{Pov}_{t}$ - is the calculated poverty rate at time or year $t$;

- $\operatorname{Pov}_{t 0}$ - is the Poverty rate at the baseline or reference year;

- $C_{i}$ - represents the intensity of impact the baseline poverty rate on future rates for each growth moment, and;

- $K_{i}$ - represents the growth rate of poverty rate for each growth moment.

Using the exponential growth function, the parameters are specified to create two optimistic and one optimistic projections (Extended Data Table 2), i.e. three deterministic time series. In the first pessimistic projection, we assume that the poverty rate grows expressively for five years and then stabilizes in subsequent years. In the second, the poverty rate grows significantly for three years and then stabilizes in subsequent years. As for the optimistic projection, poverty rate reduces expressively for five years and stabilizes in subsequent years.

Parameter estimation. All data sets used in this article are publicly available. We collect Brazilian data on the HIV/AIDS Epidemiological Bulletin of 2020 [22]. We consider in the model calibration, the annual new cases of AIDS and the annual AIDS deaths between the years 2007 and 2019. Due to the COVID-19 pandemic, we do not consider data of the year 2020. 
The demographic terms in the models (1) and (2) were estimated. The parameter $\mu$ was assumed as the inverse of the mean value of the Brazilian life expectancy between 2003 and 2030. The recruitment rate $\Lambda$ was found in order to the value of the total population from the model $N$ approximates the Brazilian population size between 2003 and 2030.

We calibrate the model (1) to the reported data of new AIDS cases and annual deaths from 2007 to 2019 (Extended Data Fig. 2), represented by $\hat{C}$ and $\hat{D}$, respectively.

We include the equations in the system (1)

$$
\begin{aligned}
\frac{d F_{1}}{d t} & =\rho I+\alpha_{2} T \\
\frac{d F_{2}}{d t} & =\delta_{1} A
\end{aligned}
$$

where $F_{1}$ represents cumulative new cases and $F_{2}$ represents cumulative annual AIDS deaths. To compare annual data, we consider

$$
\begin{aligned}
& C(i)=F_{1}(i)-F_{1}(i-1) \\
& D(i)=F_{2}(i)-F_{2}(i-1)
\end{aligned}
$$

representing the new AIDS cases and annual deaths from the model (1).

Parameters presented in Table 2 were estimated by fitting the new AIDS cases and annual AIDS deaths. We used a genetic algorithm (GA) in order to fit the model to the data. A genetic algorithm is an optimization technique in which an initial population of "chromosomes" is generated and a proportion of them may survive to the next generation or be replaced by a new individual, depending on its fitness score [58]. The fitness score is given by the mean squared errors (RMSE)

$$
\begin{aligned}
& E_{C}=\sqrt{\frac{1}{n} \sum_{i=1}^{n}[C(i)-\hat{C}(i)]^{2}} \\
& E_{D}=\sqrt{\frac{1}{n} \sum_{i=1}^{n}[D(i)-\hat{D}(i)]^{2}}
\end{aligned}
$$

In order to obtain the values of $C(i)$ and $D(i)$, we used the fourth-order Runge-Kutta method and the initial population $I(0)=2.6 \times 10^{5}, A(0)=1.5 \times 10^{5}, C(0)=38,302, R(0)=0$, $T(0)=0.4 I(0), D(0)=11,372$ and $S(0)=1.91 \times 10^{8}-I(0)-A(0)-T(0)-R(0)$. To perform the GA, we considered the package NSGA-II, which is a multiobjective genetic algorithm [24] for the Python programming language, with 1,500 generations and initial population of $1.0 \times 10^{5}$ chromosomes in order to calibrate the model with the two data sets. To define the set of values for the parameters, the one that gave the smallest joint error for the fitness score, that is, the smallest value of $E_{C}+E_{D}$, was the parameter values chosen.

For the TB model, the same methodology was applied altering the equations (21) and (22) for the following equations 


$$
\begin{aligned}
& \frac{d F_{1}}{d t}=\phi \nu P+\omega L \\
& \frac{d F_{2}}{d t}=\mu_{T} I
\end{aligned}
$$

Here, parameters presented in Table 3 were estimated by fitting the TB incidence and annual deaths between 2003 and 2019 [23] (Extended Data Fig. 33. We applied the fourth-order Runge-Kutta method to solve the system (2), and the equations (27) and (28) assuming the initial population $I(0)=78,615, L(0)=5 \times 10^{6}, P(0)=5 \times 10^{4}, C(0)=78,615, D(0)=4,987$ and $S(0)=1.7687 \times 10^{8}-I(0)-L(0)-P(0)$ in order to define the TB parameter values fitted to the data.

\section{Acknowledgments}

This study was supported by the National Institute of Allergy and Infectious Diseases (NIAID), of the US National Institute of Health (NIH), Grant Number: 1R01AI152938, and by the the Brazilian Ministry of Health (VPGDI-003-FIO19). DR acknowledges support from the Spanish Ministry of Science and Innovation and State Research Agency through the Centro de Excelencia Severo Ochoa 2019-2023 Program (CEX2018-000806-S), and support from the Generalitat de Catalunya through the CERCA Program.

\section{Competing interests}

The authors declare no competing interests. 


\section{References}

[1] Nations, U. The sustainable development goals report: 2021 (2021). URL https://unstats.un.org/sdgs/report/2021/ The-Sustainable-Development-Goals-Report-2021.pdf.

[2] World Health Organization. World health report : 2002 (2002). URL https://www.who.int/publications/i/item/9241562072.

[3] Micah, A. E. et al. Health sector spending and spending on hiv/aids, tuberculosis, and malaria, and development assistance for health: progress towards sustainable development goal 3. The Lancet 396, 693-724 (2020).

[4] Lozano, R. et al. Measuring progress from 1990 to 2017 and projecting attainment to 2030 of the health-related sustainable development goals for 195 countries and territories: a systematic analysis for the global burden of disease study 2017. The Lancet 392, 2091-2138 (2018).

[5] World Health Organization. The End TB Strategy (2015). URL https: //www.who.int/teams/global-tuberculosis-programme/ the-end-tb-strategy.

[6] Carter, D. J. et al. The impact of social protection and poverty elimination on global tuberculosis incidence: a statistical modelling analysis of sustainable development goal 1. The Lancet Global Health 6, e514-e522 (2018).

[7] Taaffe, J., Cheikh, N. \& Wilson, D. The use of cash transfers for hiv prevention-are we there yet? African Journal of AIDS Research 15, 17-25 (2016).

[8] Stoner, M. C., Kilburn, K., Godfrey-Faussett, P., Ghys, P. \& Pettifor, A. E. Cash transfers for hiv prevention: A systematic review. PLoS medicine 18, e1003866 (2021).

[9] Hopkins, J. Brazil - COVID-19 Overview (2020). URL https:// coronavirus.jhu.edu/region/brazil.

[10] de Arruda, P. L., de Andrade, M. L., Falcão, T., Barbosa, D. T. \& Morgandi, M. Auxílio emergencial - lessons from the brazilian experience responding to covid-19 (2021). URL https://documents1. 
worldbank.org/curated/en/099255012142121495/pdf / P1748361b302ee5718913146b11956610692e4faf5bc.pdf.

[11] Rasella, D., Aquino, R., Santos, C. A., Paes-Sousa, R. \& Barreto, M. L. Effect of a conditional cash transfer programme on childhood mortality: a nationwide analysis of brazilian municipalities. The lancet 382, 57-64 (2013).

[12] Rasella, D. et al. Child morbidity and mortality associated with alternative policy responses to the economic crisis in brazil: A nationwide microsimulation study. PLoS medicine 15, e1002570 (2018).

[13] Hone, T. et al. Effect of economic recession and impact of health and social protection expenditures on adult mortality: a longitudinal analysis of 5565 brazilian municipalities. The Lancet Global Health 7, e1575-e1583 (2019).

[14] Trajman, A., Saraceni, V. \& Durovni, B. Sustainable development goals and tuberculosis in brazil: challenges and potentialities. Cadernos de saude publica 34, e00030318 (2018).

[15] Ministério da Saúde. Boletim Epidemiológico HIV/Aids - 2021 (2021). URL http://www.aids.gov.br/pt-br/pub/2021/ boletim-epidemiologico-hivaids-2021.

[16] Silva, C. J. \& Torres, D. F. A sica compartmental model in epidemiology with application to hiv/aids in cape verde. Ecological complexity 30, 70-75 (2017).

[17] Omondi, E., Mbogo, R. \& Luboobi, L. A mathematical modelling study of hiv infection in two heterosexual age groups in kenya. Infectious Disease Modelling 4, 83-98 (2019).

[18] Trauer, J. M., Denholm, J. T. \& McBryde, E. S. Construction of a mathematical model for tuberculosis transmission in highly endemic regions of the asia-pacific. Journal of theoretical biology 358, 74-84 (2014).

[19] Huo, H.-F., Chen, R. \& Wang, X.-Y. Modelling and stability of hiv/aids epidemic model with treatment. Applied Mathematical Modelling 40, 65506559 (2016).

[20] Gomes, M. G. M. et al. Introducing risk inequality metrics in tuberculosis policy development. Nature Communications 10, 1-12 (2019). 
[21] Galanis, G. \& Hanieh, A. Incorporating social determinants of health into modelling of covid-19 and other infectious diseases: A baseline socioeconomic compartmental model. Social science \& medicine 274, 113794 (2021).

[22] Ministério da Saúde. Boletim Epidemiológico HIV/Aids - 2020 (2020). URL http://www.aids.gov.br/pt-br/pub/2020/ boletim-epidemiologico-hivaids-2020.

[23] Ministério da Saúde. Banco de dados do Sistema Único de Saúde - DATASUS (1991). URL http: / /www2 . datasus.gov.br/.

[24] Deb, K., Pratap, A., Agarwal, S. \& Meyarivan, T. A fast and elitist multiobjective genetic algorithm: Nsga-ii. IEEE Transactions on Evolutionary Computation 6, 182-197 (2002).

[25] Instituto Brasileiro de Geografia e Estatística. Projeção da população do brasil e das unidades da federação (2021). URL https: / / www .ibge. gov.br/apps/populacao/projecao/index.html.

[26] World Bank. Poverty and shared prosperity 2020: Reversals of fortune (The World Bank, 2020).

[27] Ravallion, M. A comparative perspective on poverty reduction in Brazil, China, and India. World Bank Research Observer 26 (2011).

[28] Sotomayor, O. J. Can the minimum wage reduce poverty and inequality in the developing world? evidence from Brazil. World Development 138 (2021).

[29] Neri, M. C. Qual foi o impacto da crise sobre a pobreza e a distribuição de renda? Tech. Rep., FGV Social - Centro de Políticas Sociais (2018).

[30] Menezes-Filho, N., Komatsu, B. \& Rosa, J. Reducing poverty and inequality during the coronavirus outbreak: The emergency aid transfers in Brazil. Insper - Policy Paper 54 (2021).

[31] Instituto Brasileiro de Geografia e Estatística. Síntese de indicadores sociais: Uma análise das condições de vida da população brasileira 2021. Tech. Rep., Institute of Applied Economic Research (IPEA) - n. 1647 (2021). 
[32] Oliosi, J. G. N. et al. Effect of the bolsa familia programme on the outcome of tuberculosis treatment: a prospective cohort study. The Lancet global health 7, e219-e226 (2019).

[33] Torrens, A. W. et al. Effectiveness of a conditional cash transfer programme on tb cure rate: a retrospective cohort study in brazil. Transactions of the Royal Society of Tropical Medicine and Hygiene 110, 199-206 (2016).

[34] Lin, H.-H., Ezzati, M. \& Murray, M. Tobacco smoke, indoor air pollution and tuberculosis: a systematic review and meta-analysis. PLoS medicine 4, e20 (2007).

[35] Joy, R. et al. Impact of neighborhood-level socioeconomic status on hiv disease progression in a universal health care setting. JAIDS Journal of Acquired Immune Deficiency Syndromes 47, 500-505 (2008).

[36] Maruthappu, M., Zhou, C., Williams, C., Zeltner, T. \& Atun, R. Unemployment and hiv mortality in the countries of the organisation for economic cooperation and development: 1981-2009. JRSM open 8, 2054270416685206 (2017).

[37] Feleke, B. E., Feleke, T. E. \& Biadglegne, F. Nutritional status of tuberculosis patients, a comparative cross-sectional study. BMC pulmonary medicine 19, 1-9 (2019).

[38] McMahan, L. D. et al. Getting to zero hiv/aids in sub-saharan africa: Understanding perceptions of locals using the social determinants of health framework. Health \& Social Care in the Community (2021).

[39] Burch, L. S., Smith, C. J., Phillips, A. N., Johnson, M. A. \& Lampe, F. C. Socioeconomic status and response to antiretroviral therapy in high-income countries: a literature review. Aids 30, 1147-1162 (2016).

[40] Pettifor, A. et al. The effect of a conditional cash transfer on hiv incidence in young women in rural south africa (hptn 068): a phase 3, randomised controlled trial. The Lancet Global Health 4, e978-e988 (2016).

[41] Stoner, M. C. et al. The effect of school attendance and school dropout on incident hiv and hsv-2 among young women in rural south africa enrolled in hptn 068. AIDS (London, England) 31, 2127 (2017). 
[42] Huppert, A. \& Katriel, G. Mathematical modelling and prediction in infectious disease epidemiology. Clinical microbiology and infection 19, 9991005 (2013).

[43] Ministério da Saúde. Boletim Epidemiológico de Tuberculose - 2020 (2020). URL http://www.aids.gov.br/pt-br/pub/2020/ boletim-epidemiologico-de-turbeculose-2020.

[44] World Health Organization. Hiv/aids (2021). URL https : / / www . who . int/news-room/questions-and-answers/item/hiv-aids.

[45] Ali, J. H. \& Yirtaw, T. G. Time to viral load suppression and its associated factors in cohort of patients taking antiretroviral treatment in east shewa zone, oromiya, ethiopia, 2018. BMC infectious diseases 19, 1-6 (2019).

[46] Leng, X. et al. Hiv virological failure and drug resistance among injecting drug users receiving first-line art in china. BMJ open 4, e005886 (2014).

[47] Jobanputra, K. et al. Factors associated with virological failure and suppression after enhanced adherence counselling, in children, adolescents and adults on antiretroviral therapy for hiv in swaziland. PloS one 10, e0116144 (2015).

[48] Hassan, A. S. et al. Hiv-1 virologic failure and acquired drug resistance among first-line antiretroviral experienced adults at a rural hiv clinic in coastal kenya: a cross-sectional study. AIDS research and therapy 11, 112 (2014).

[49] Rupérez, M. et al. Determinants of virological failure and antiretroviral drug resistance in mozambique. Journal of Antimicrobial Chemotherapy 70, 2639-2647 (2015).

[50] Todd, J. et al. Time from hiv seroconversion to death: a collaborative analysis of eight studies in six low and middle-income countries before highly active antiretroviral therapy. AIDS (London, England) 21, S55 (2007).

[51] Centers for Disease Control and Prevention. Evidence of hiv treatment and viral suppression in preventing the sexual transmission of hiv (2020). URL https://www.cdc.gov/hiv/pdf/risk/art/ cdc-hiv-art-viral-suppression.pdf. 
[52] Haley, C. A. Treatment of latent tuberculosis infection. Microbiology spectrum 5, 5-2 (2017).

[53] Tang, P. \& Johnston, J. Treatment of latent tuberculosis infection. Current treatment options in infectious diseases 9, 371-379 (2017).

[54] Garziera, G. et al. Latent tuberculosis infection and tuberculosis in patients with rheumatic diseases treated with anti-tumor necrosis factor agents. Clinical rheumatology 36, 1891-1896 (2017).

[55] Mack, U. et al. Ltbi: latent tuberculosis infection or lasting immune responses to m. tuberculosis? a tbnet consensus statement. European Respiratory Journal 33, 956-973 (2009).

[56] World Health Organization. Global tuberculosis report 2018 (World Health Organization, 2018).

[57] Lütkepohl, H. New introduction to multiple time series analysis (Springer Science \& Business Media, 2005).

[58] Whitley, D. A genetic algorithm tutorial. Statistics and computing 4, 65-85 (1994). 
Extended Data Table 1 Fitted parameters of the HIV/AIDS and TB models. Parameter values obtained by the multiobjective genetic algorithm in order to fit to the TB and AIDS incidence and deaths.

\begin{tabular}{ll|ll}
\hline \multicolumn{4}{c}{ HIV/AIDS model } \\
\hline Parameter & Fitted Value & Parameter & Fitted Value \\
\hline$\beta_{H 1}$ & 0.0487 & $\beta_{H 2}$ & 4.5235 \\
$\delta_{11}$ & 0.0738 & $\delta_{12}$ & 0.0279 \\
$\delta_{12}$ & 0.0176 & $\delta_{22}$ & 0.0465 \\
$\alpha_{11}$ & 0.3452 & $\alpha_{12}$ & 0.1245 \\
$\alpha_{22}$ & 0.0941 & $\alpha_{22}$ & 0.0011 \\
$\rho_{1}$ & 0.0885 & $\rho_{2}$ & 0.0697 \\
$\eta_{1}$ & 3.3376 & $\eta_{2}$ & 3.7288 \\
$\gamma_{1}$ & 0.2116 & $\gamma_{2}$ & 0.3766 \\
$\varepsilon_{11}$ & 0.0294 & $\varepsilon_{12}$ & 0.0296 \\
$\varepsilon_{12}$ & 0.0007 & $\varepsilon_{22}$ & 0.0031 \\
\hline
\end{tabular}

\begin{tabular}{ll|ll}
\hline Parameter & Fitted Value & Parameter & Fitted Value \\
\hline$\beta_{T 1}$ & 3.0939 & $\beta_{T 2}$ & 2.9195 \\
$\nu_{1}$ & 2.9316 & $\nu_{2}$ & 2.6039 \\
$\tau_{1}$ & 0.9624 & $\tau_{2}$ & 0.8349 \\
$\omega_{1}$ & 0.0093 & $\omega_{2}$ & 0.0161 \\
$\mu_{T 1}$ & 0.0557 & $\mu_{T 2}$ & 0.0315 \\
$\phi$ & 0.0203 & $\theta$ & 0.7633 \\
$\sigma$ & 0.0059 & & \\
\hline
\end{tabular}

Extended Data Table 2 Parameter specification for the exponential growth function.

\begin{tabular}{lrrr}
\hline & & \multicolumn{2}{c}{ Scenarios } \\
\cline { 3 - 4 } & & Optimistic & Pessimistic \\
\hline \multirow{2}{*}{ Growth moment 1 } & $K_{1}$ & 1.2 & 1.9 \\
& $C_{1}$ & 0.9 & 0.8 \\
\hline \multirow{2}{*}{ Growth moment 2 } & $K_{2}$ & 0.02 & 0.02 \\
& $C_{2}$ & 1.5 & 2.0 \\
\hline
\end{tabular}

Note: The parameters are chosen such that the the predicted values for $P_{0 v_{2020}}$ are closest to the real values. 


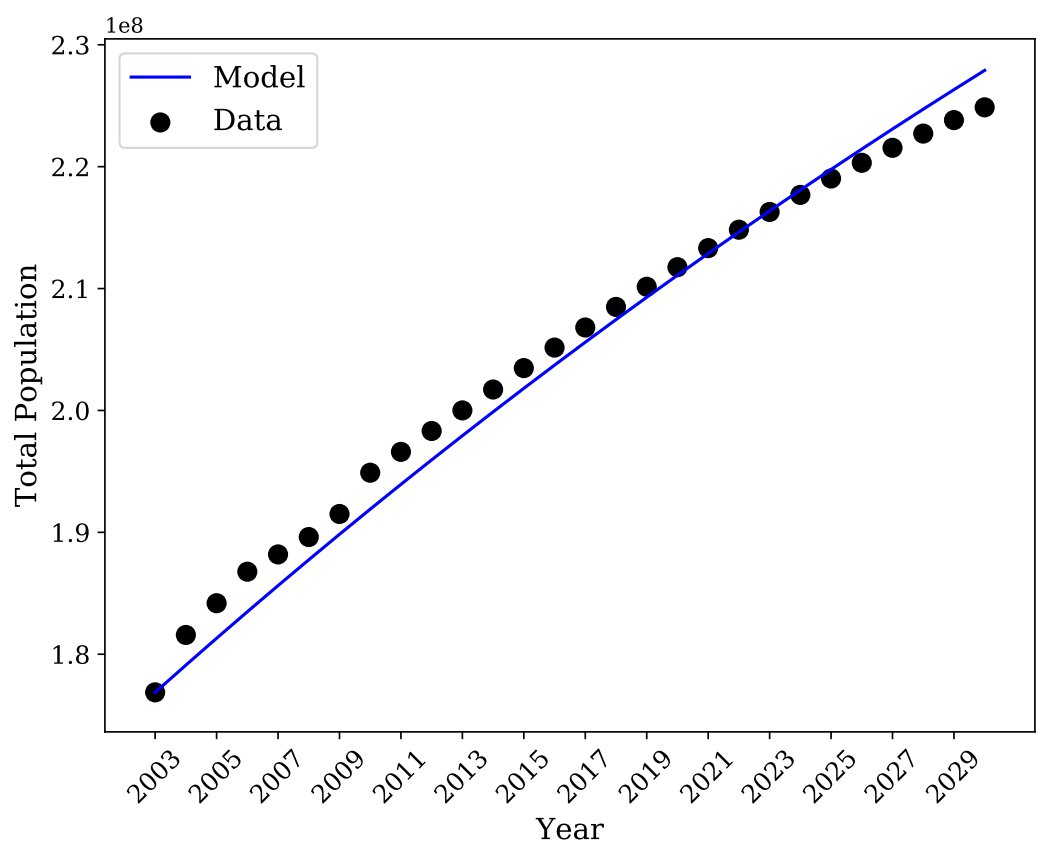

Extended Data Fig. 1 Function representing the Brazilian total population fitting to the data and population projection until 2030 reported by Health Minister, in which the birth rate of the HIV/AIDS and TB models was estimated. 

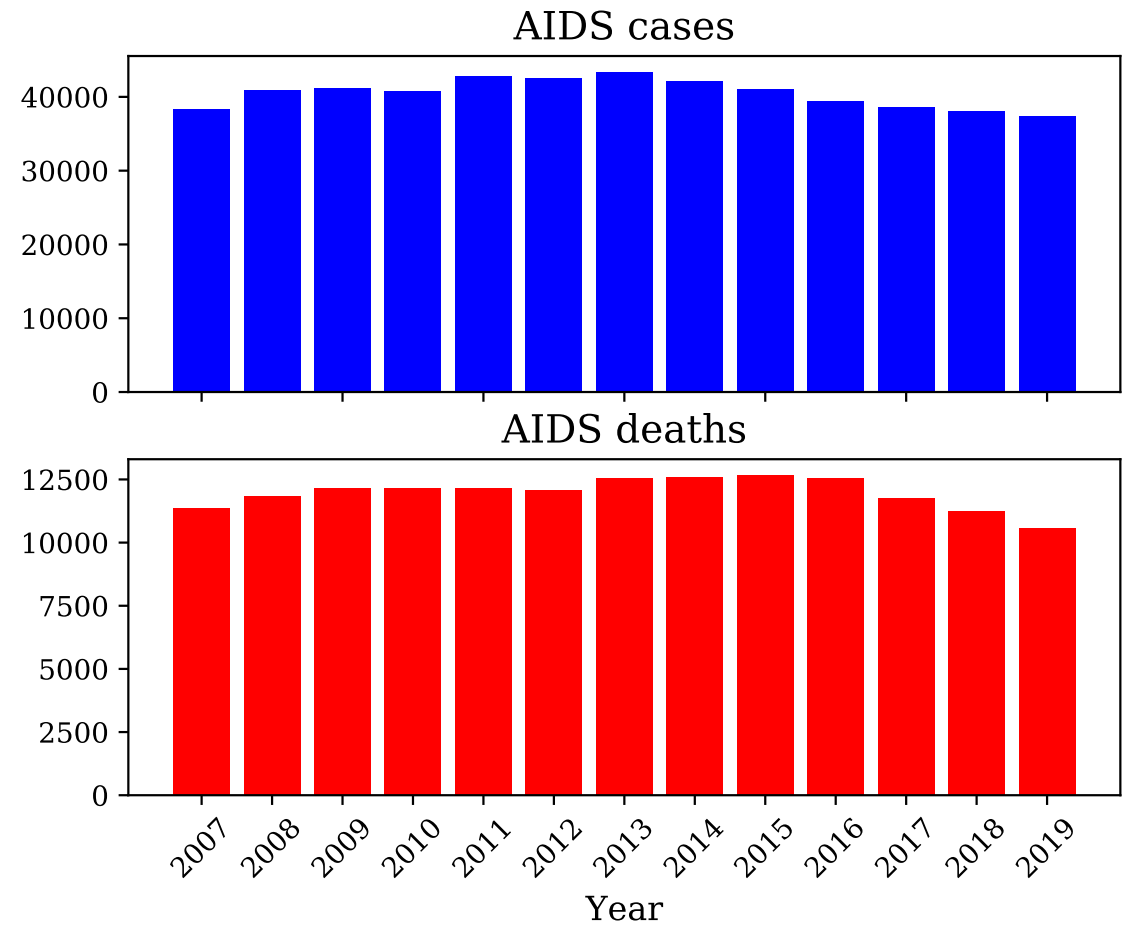

Extended Data Fig. 2 Annual AIDS new cases and deaths between 2007 and 2019. 

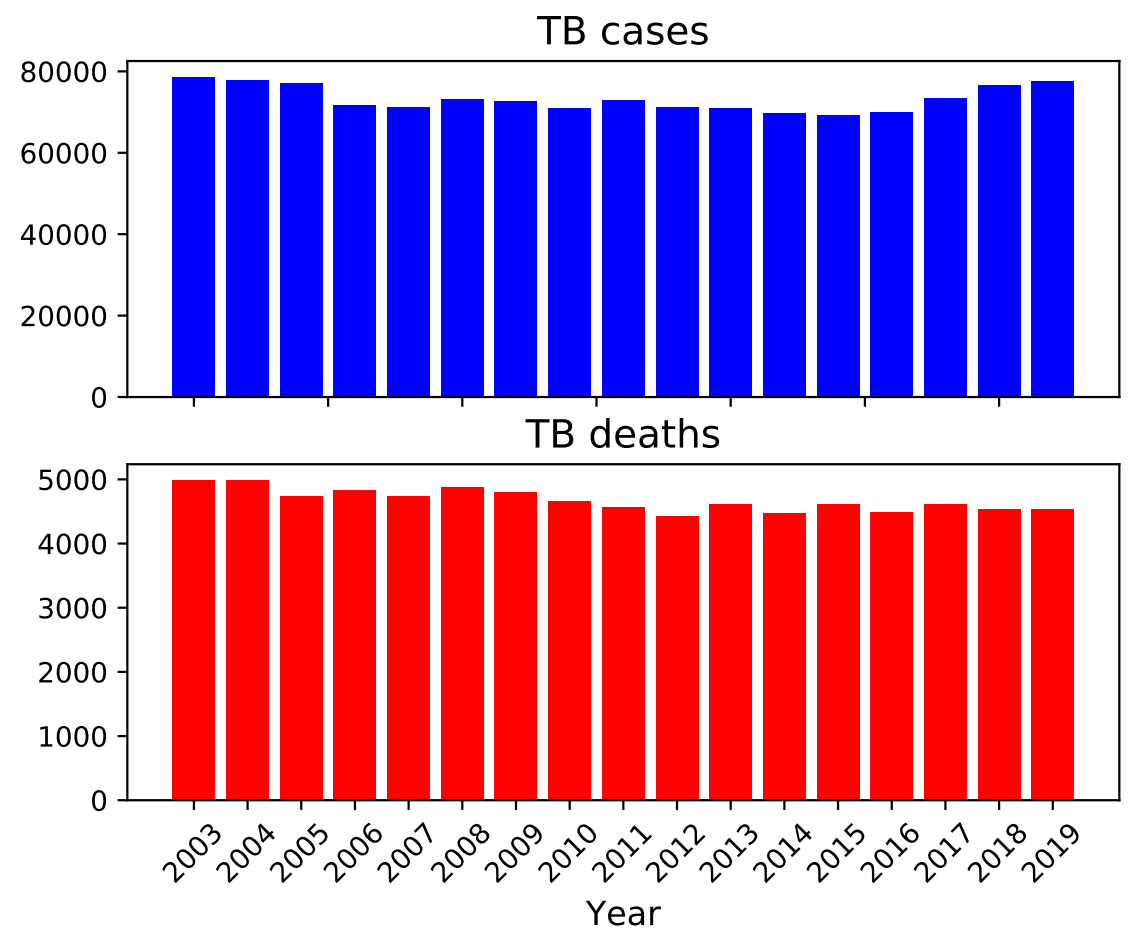

Extended Data Fig. 3 Annual TB new cases and deaths between 2003 and 2019. 


\section{Supplementary Files}

This is a list of supplementary files associated with this preprint. Click to download.

- Supplementary.pdf

- flatRasellars.pdf

- flatRasellaepc.pdf 